Several serological biomarkers were measured in each cohort, selected due to the specific tissue metabolite they represent. These included: C2M (cartilage degradation); CTX-I and PINP (bone resorption and formation); C1M and C3M (interstitial matrix degradation); CRPM (CRP metabolite) and VICM (macrophage activity).

Each biomarker was log transformed and min-max normalised in order to allow for direct comparison of each of the variables. Patient clustering was performed using Ward hierarchical clustering and the number of clusters determined using the GAP statistic. ANOVA test was used to identify differences in delta change in radiographic scores at 24 and 52 weeks in the $R A$ placebo groups $(n=271)$ only.

Results: Clustering analysis resulted in five different clusters $(A-E)$. Cluster $A$ and $B$ were both comprised of $>98 \%$ RA patients. Cluster $D$ was comprised mainly of OA patients whilst clusters $\mathrm{C}$ and $\mathrm{E}$ were a mix of $\mathrm{OA}$ and $\mathrm{RA}$ patients.

Clusters $\mathrm{A}$ and $\mathrm{B}$ were characterised by high levels of all biomarkers compared to other clusters except for VICM, which is significantly lower in cluster $A$ than in cluster $B$ (Tukey test $p<0.001$ ). Biomarker levels in Cluster $C$ were all close to the median. Cluster D was characterised by low levels of all biomarkers compared to other clusters with significantly lower $\mathrm{C} 2 \mathrm{M}$ levels, whilst cluster $\mathrm{E}$ also had low levels of markers, yet with significantly higher levels of CTX-1 compared to cluster D. When looking at the RA placebo groups there were no difference in change in SHP score at 24 weeks between the groups, $(n=271$, LITHE, OSKIRA), but a significant difference in SHP change 52 weeks $(n=83, p<0.05$, LITHE).

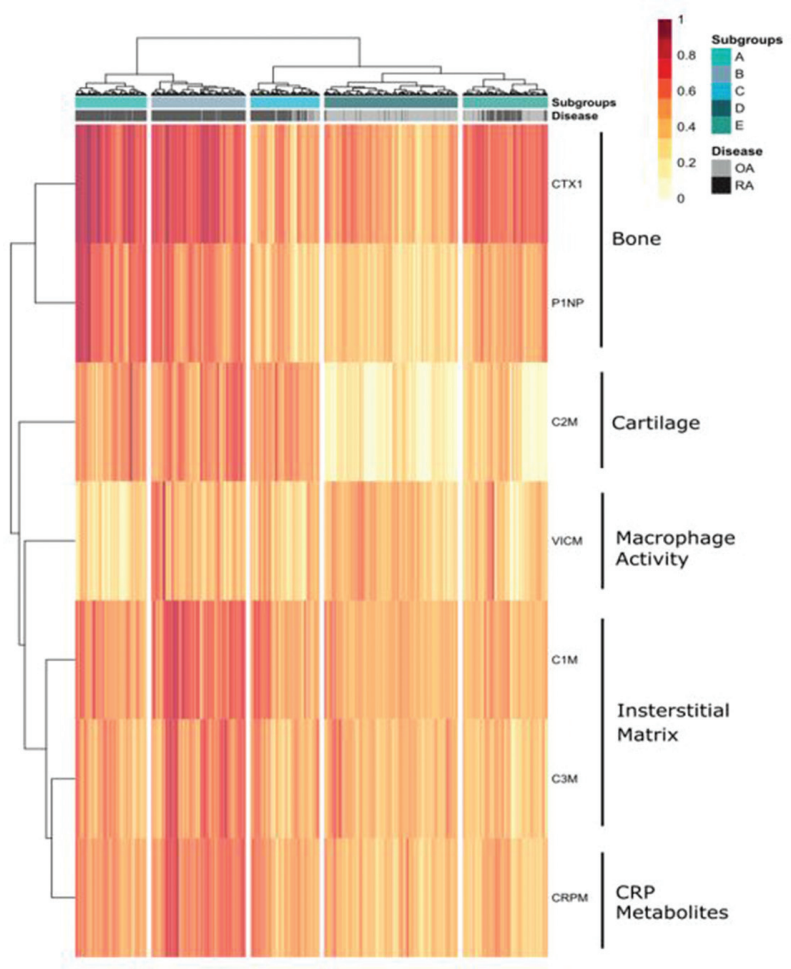

Abstract AB0250 - Figure 1

Conclusions: We have identified putative RA profiles based on novel serological biomarker status. Whether patients in particular clusters may benefit from specific targeted treatments, according to their tissue turnover profile, will be investigated further.

Disclosure of Interest: None declared

DOI: 10.1136/annrheumdis-2018-eular.3071

\section{AB0251 ARE ACPA ASSOCIATED WITH MORE BONE LOSS OVER TIME IN PATIENTS WITH RA?}

J.A.M.P. Amkreutz ${ }^{1}$, E.C. de Moel ${ }^{1}$, L. Heimans ${ }^{2}$, C.F. Allaart ${ }^{1}$, T.W.J. Huizinga ${ }^{1}$, D. van der Woude ${ }^{1} .{ }^{1}$ Rheumatology; ${ }^{2}$ Internal Medicine, Leiden University Medical Center, Leiden, Netherlands

Background: Anti-citrullinated protein antibodies (ACPA) are one of the most important serological markers for rheumatoid arthritis (RA) and have been suggested to play a pathophysiologic role by directly binding to osteoclasts. However, the effect of ACPA on systemic bone mineral density (BMD) and in particular their effect on changes in BMD over time is currently unknown.
Objectives: The aim of this study was to determine whether ACPA associate with changes in BMD over time in patients with RA.

Methods: Yearly dual X-ray absorptiometry (DXA) scores were performed during 5 years of follow-up in 412 patients with recent-onset RA participating in the IMPROVED study ${ }^{1}$, a clinical trial in which patients were treated according to a remission- (disease activity score <1.6) steered strategy. The effect of the pres ence of ACPA on 1) Z-scores of lumbar spine and hip over time, and 2) prevalence of osteopenia/osteoporosis (defined as a T-score $\leq-1$ ) over time was analysed using generalised estimating equations. Analyses were adjusted for age, gender, BMI, symptom duration, smoking status, disease activity, prednisone intake, usage of bisphosphonates, calcium intake and serum 25-OH vitamin D levels.

Results: ACPA-positive patients had a significantly lower lumbar spine $(p=0.04$ and hip $(\mathrm{p}=0.01) \mathrm{Z}$-score at baseline. There was no difference in prevalence of osteoporosis/osteopenia at baseline between ACPA-positive and ACPA-negative patients (OR $(95 \% \mathrm{Cl}) 1.02(0.55$ to 1.19$))$. We hypothesised that ACPA-positive patients would have more BMD loss over time compared to ACPA-negative patients. However, ACPA-positivity did not associate with a stronger decline in Z score over time at lumbar $(p=0.43$ ) or femoral sites $(p=0.67)$. Additionally, no effect of ACPA-positivity was found on the development of osteoporosis/osteopenia over time $(p=0.23)$

Conclusions: ACPA-positive patients have a significantly lower baseline BMD compared to ACPA-negative patients. Surprisingly, ACPA do not associate with a decrease in BMD over time in patients who were treated according to a tight control strategy. These results indicate that ACPA alone do not contribute to bone loss after disease onset in the absence of inflammation/disease activity.

\section{REFERENCE:}

[1] Wevers-de Boer K, et al. Ann Rheum Dis. 2012 Sep;71(9):1472-7.

Disclosure of Interest: None declared

DOI: 10.1136/annrheumdis-2018-eular.6301

\section{$\mathrm{AB} 0252$ \\ LOOSING DAS28-ESR, BUT STAYING IN BOOLEAN REMISSION- IS IT POSSIBLE? DATA FROM THE PROSPECTIVE, RANDOMISED RETRO TRIAL ON RHEUMATOID ARTHRITIS PATIENTS IN STABLE} REMISSION

J. Erhard ${ }^{1}$, M. Hagen ${ }^{1}$, M. Englbrecht ${ }^{1}$, J. Haschka ${ }^{2}$, M. Reiser ${ }^{1}$, A. Kleyer ${ }^{1}$, A. J. Hueber ${ }^{1}$, B. Manger ${ }^{1}$, C. Figueiredo ${ }^{3}$, J.F. Cobra ${ }^{3}$, H.-P. Tony ${ }^{4}$, S. Finzel ${ }^{5}$, S. Kleinert ${ }^{6}$, J. Wendler ${ }^{7}$, F. Schuch ${ }^{7}$, M. Ronneberger ${ }^{7}$, M. Feuchtenberger ${ }^{8}$ M. Fleck ${ }^{9}$, K. Manger ${ }^{10}$, W. Ochs ${ }^{11}$, M. Schmitt-Haendle ${ }^{11}$, H. Lorenz ${ }^{12}$, H. Nuesslein ${ }^{13}$, R. Alten ${ }^{14}$, J. Henes ${ }^{15}$, K. Krueger ${ }^{16}$, G. Schett ${ }^{1}$, J. Rech ${ }^{1}$. ${ }^{1}$ Department of Internal Medicine 3, Rheumatology an, University of Erlangennuremberg, Erlangen, Germany; ${ }^{2}$ St. Vincent Hospital, Vinforce Study Group, Medical University Vienna, Vienna, Austria; ${ }^{3}$ Insitutio de Rheumatologia, Sao Paolo, Brazil; ${ }^{4}$ University of Wuerzburg, Internal Medicine 2, Wuerzburg; ${ }^{5}$ University Medical Center Freiburg, Rheumatology and Clinical Immunology, Freiburg; ${ }^{6}$ Rheumatology Clinical Practice; ${ }^{7}$ Rheumatology Clinical Practice Erlangen, Erlangen; ${ }^{8}$ Rheumatology Practice and Department of Internal Medicine 2. Clinic Burghausen, Burghausen; ${ }^{9}$ Department of Rheumatology and Clinical Immunology, Asklepios Medical Center, Bad Abbach; ${ }^{10}$ Rheumatology Practice Bamberg, Bamberg: ${ }^{11}$ Rheumatology Practice Bayreuth, Bayreuth; ${ }^{12}$ University of Heidelberg, Medicine 5, Heidelberg; ${ }^{13}$ Rheumatology Practice Nuernberg, Nuernberg: ${ }^{14}$ Schlosspark Klinik, Internal Medicine/Rheumatology, Berlin; ${ }^{15}$ University of Tuebingen, Centre for Interdisciplinary Clinical Immunology, Tuebingen; ${ }^{16}$ Praxiszentrum St. Bonifatius, Munich, Germany

Background: DAS28-ESR is the most widely used instrument to assess remission in rheumatoid arthritis (RA) patients. Nonetheless, substantial residual dis ease activity can be present in RA patients fullfilling DAS28-ESR remission. Therefore, more stringent criteria for remission have been developed. While it is known that patients can fulfil DAS28-ESR but fail ACR/EULAR Boolean remission criteria, the existence of the reverse is less known.

Objectives: To test the possibility to loose DAS28-ESR remission while staying in ACR/EULAR remission in patients with RA

Methods: Data were obtained from the prospective randomised RETRO study (EudraCT: 2009-015740-42), which recruits RA patients in stable remission. Remission was assessed by the following instruments every three months: DAS28-ESR, DAS28-CRP, CDAI, SDAI, PAS, and ACR/EULAR criteria. In the group of patients, escaping DAS28-ESR remission, but fulfilling ACR/EULAR Boolean remission, the individual components of DAS28-ESR were analysed that determined their escape from remission.

Results: 142 patients analysed, which were all in DAS28-ESR remission at baseline. Of them, 140 (98.59\%) were in DAS-CRP-remission, $131(92.25 \%)$ in CDAlremission, 130 (91.55\%) in SDAl-remission, 109 (76.76\%) in ACR/EULAR remission and 66 (46.48\%) in PAS-remission. We analysed upon the 1 year follow up 
those patients loosing DAS28-ESR remission over time: 58 patients lost DASESR remission at least once during the 4 follow-up visits. Surprisingly, 24\% (3 months), $36 \%$ (6 months), $24 \%$ (9 months) and $28 \%$ (12 months) of the patients still fulfilled the Boolean remission criteria. The only plausible reason for failing DAS28-ESR remission but staying in ACR/EULAR remission is an isolated elevation of the ESR, not accompanied by increased signs and symptoms of disease. Indeed all patients loosing DAS28-ESR remission but staying in ACR/EULAR Boolean remission had an elevated ESR equal or higher than $15 \mathrm{~mm}$. However, if DAS28 scores were calculated by C-reactive protein in the same patients, they all fulfilled remission criteria.

Conclusions: DAS28-ESR remission can be missed even if a patient fulfils the more stringent ACR/EULAR Boolean remission criteria. The reason for this remarkable constellation is an elevated erythrocyte sedimentation rate without any clinical symptoms. Hence, isolated elevations of erythrocyte sedimentation rate should be seen critical. These data show the limitations of individual instruments to assess remission in RA and show that interpretations of the erythrocyte sedimentation rate need to be done in the clinical context

Disclosure of Interest: None declared

DOI: 10.1136/annrheumdis-2018-eular.5521

\section{AB0253 RAPID REMISSION DURING THE FIRST YEAR IN EARLY ACTIVE RHEUMATOID ARTHRITIS IS ASSOCIATED WITH BETTER 5 YEARS STRUCTURAL DAMAGE OUTCOMES}

J. Legrand ${ }^{1}$, T. Kirchgesner ${ }^{1}$, T. Sokolova ${ }^{2}$, B. Vande Berg ${ }^{1}$, P. Durez ${ }^{2}$.

${ }^{1}$ Musculoskeletal Imaging Unit, ${ }^{2}$ Rheumatology, Cliniques universitaires Saint-Luc - Université Catholique de Louvain - Institut de Recherche Expérimentale et Clinique (IREC), Brussels, Belgium

Background: Remission is arguably the best and ultimate therapeutic goal in early rheumatoid arthritis (ERA). ERA patients who reach rapid and sustained remission are likely to retain good radiographic outcomes.

Objectives: The aim of this study was to evaluate the radiographic progression and the newly pathological joints in a Belgian ERA cohort during a 5 to 15 years follow-up and to correlate the Sharp score index with different indices of clinical remission observed during the first year.

Methods: This was a retrospective analysis of mean $(95 \% \mathrm{Cl})$ change from baseline $(B L)$ to 5 years Sharp/Van der Heijde (SVdH) scoring according to DAS28, SDAI, CDAI and ACR/EULAR Boolean remission scores for patients at Month 3, 6 and 12. Newly pathological joint defined by a SVdH score of 0 at baseline and a score greater than or equal to 1 at follow up were analysed as a secondary objective. 133 ERA patients naïve to DMARDs therapies were analysed (100 women and 33 men) with a mean age $\pm S D(49.9 \pm 13.3)$, mean DAS 28-CRP $(4.89 \pm 1.3)$, mean HAQ (1.25 \pm 0.67$)$, mean SDAI (28.4 \pm 15.5$)$, mean CDAI $(25.8 \pm 14.8)$ and mean SVdH (6 \pm 14$)$. Differences were statistically tested using t tests.

Results: ERA patients were divided in 2 groups: "Xrays Stable" if the delta of SVdH score was $<10(n=90)$ or "Xrays Progressive" if the delta of SVdH score was $>10(n=43)$. As expected, presence of ACPA and baseline erosion were significantly higher in the Xrays progressive group. No significant baseline characteristics differences were observed for DAS28-CRP, CDAI, SDAI, HAQ. smoking status, swollen joint count or CRP.\% of patients reaching DAS 28CRP, SDAI, $\mathrm{CDAl}$ and Boolean remission rates observed at 6 months were statistically significant different between groups (fig). There was no significant difference in clinical responses between subgroups of patients who developed new pathological joints and those who did not.

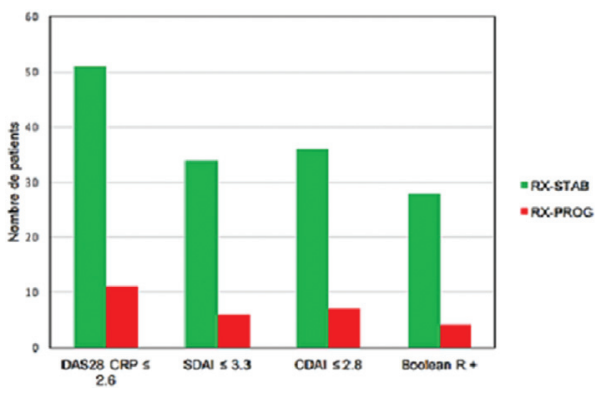

Abstract AB0253 - Figure 1

Conclusions: These results demonstrate that remission is an important therapeutic goal to protect joint damage in ERA. All remission criteria were able to predict the radiological prevention. The identification of a new pathologic joint is not associated with lack of response.
Disclosure of Interest: None declared

DOI: 10.1136/annrheumdis-2018-eular.4940

\section{AB0254 THE IMPACT OF THE PATIENT GLOBAL ASSESSMENT VARIATION ON THE DAS 28 VALUE}

K. ben abdelghani, M. boudokhane, M. chammakhi, A. fazaa, K. ouenniche,

S. kassab, S. chekili, A. laatar. Rheumatology, - Mongi Slim Hospital - La MarsaTunisia, Marsa, Tunisia

Background: Rheumatoid arthritis (RA) is the most frequent chronic inflammatory rheumatism. The DAS 28 is a disease activity measure method used to assess RA activity. It is a composite score taking into account 4 items: the number of swollen joints/28, the number of tender joints/28, the erythrocyte sedimentation rate $(E S R)$ or $C$ reactive protein (CRP) rate, the patient global assessment (PGA) indicated on a $0-10 \mathrm{~cm}$ visual analogue scale (VAS) with 'not active at all' and 'extremely active' as anchors. The DAS28 determination is very important since it guides the therapeutic decision.

Objectives: The aim of this study was to determine the different ways of asking about the PGA and to assess the impact of its value variation on the calculation of the DAS 28.

Methods: In order to determine how to evaluate the GPA, a questionnaire including 4 propositions was asked to a cohort of Tunisian rheumatologists:

1. how do you assess your health status this past week?

2. what is the degree of the disease impact in your life this last week?

3. what is the degree of the disease activity this last week?

4. other

Then, a DAS 28 calculation was proceeded according to the different choices of GPA question method for 10 Tunisian patients.

Results: The questionnaire was proposed to 37 rheumatologists, 15 working in the private sector and 22 in the public sector. These latter were 9 assistants, 3 professors, 5 specialist doctors and 5 associate professors. The first, second, third and fourth propositions were respectively chosen by 2, 14, 19 and 2 physicians. Subsequently DAS 28 was calculated. In the table 1 below, the variation of the DAS 28 value according to the choice of the PGA method is shown:

\begin{tabular}{lcccc}
\multicolumn{1}{l}{ Abstract AB0254 - Table 1 } \\
\hline Patient & $\begin{array}{c}\text { DAS 28 } \\
\text { (PGA1) }\end{array}$ & $\begin{array}{c}\text { DAS 28 } \\
\text { (PGA2) }\end{array}$ & $\begin{array}{c}\text { DAS 28 } \\
\text { (PGA3) }\end{array}$ & $\begin{array}{c}\text { DeltaDAS 28(DAS28 max- } \\
\text { min) }\end{array}$ \\
\hline 1 & 2.95 & 2.95 & 2.81 & 0.14 \\
2 & 2.53 & 2.67 & 2.67 & 0.14 \\
3 & 2.1 & 1.96 & 1.96 & 0.14 \\
4 & 5.82 & 5.82 & 5.54 & 0.28 \\
5 & 5.30 & 5.30 & 5.16 & 0.14 \\
6 & 2.87 & 2.87 & 2.87 & 0.00 \\
7 & 8.22 & 8.22 & 8.22 & 0.00 \\
8 & 4.97 & 5.11 & 5.11 & 0.14 \\
9 & 7.49 & 7.35 & 7.63 & 0.14 \\
10 & 2.03 & 2.17 & 1.89 & 0.28 \\
\hline
\end{tabular}

Conclusions: The GPA question is a subjective item taken into account for the calculation of the DAS 28. Despite the different ways of asking about it, our study showed that this factor have no real impact on the DAS28 value variation since it doesn't exceed 0.6. DAS28 remains a reliable tool in the clinical practice. Disclosure of Interest: None declared DOI: 10.1136/annrheumdis-2018-eular.5691

\section{AB0255 \\ DOES MENOPAUSE AFFECT DISEASE ACTIVITY OF RHEUMATOID ARTHRITIS(RA)? AN ANALYSIS FROM THE NINJA COHORT IN 2016}

K. Nagahata ${ }^{1}$, S. Tsuzuki ${ }^{1}$, S. Hattori ${ }^{1}$, K. Yomono $^{1}$, S. Suzuki ${ }^{1}$, S. Takenouchi ${ }^{1}$, R. Yokochi ${ }^{1}$, N. Hagino ${ }^{1}$, T. Matsui ${ }^{2}$, S. Tohma ${ }^{3} .{ }^{1}$ Division of Hematology and Rheumatology, Teikyo University Chiba Medical Center, Chiba; ${ }^{2}$ Department of Rheumatology, Clinical Research Center for Allergy and Rheumatology, National Hospital Organization Sagamihara National Hospital, Sagamihara; ${ }^{3}$ Department of Rheumatology, National Hospital Organization Tokyo National Hospital, Tokyo, Japan

Background: Early menopause may be a risk of developing $\mathrm{RA}^{1}$. Although there are many reports about menopause and the onset of RA, it is not yet clear how the disease activity of RA differs for each generation including menopause. 